\title{
Posterior association cortex lesions in rats: Mazes, pattern discrimination, and reversal learning
}

\author{
MARY G. BOYD and ROGER K. THOMAS \\ University of Georgia, Athens, Georgia 30601
}

\begin{abstract}
Lesions in either the proposed parietal or temporal cortex resulted in a dissociation of deficits, with the former affecting maze performances and "complex" but not "simple" pattern discrimination and the latter affecting performances on a series of reversals and the simple but not the complex pattern discrimination. Discussion considered the role of parietal cortex in spatial behaviors, the adequacy of the simple and complex patterns to differentiate simple sensory from integrative functions, and the unqualified use of Lashley's principles of mass function and equipotentiality. It was also suggested that the rat might provide a useful model for investigations of posterior association cortex.
\end{abstract}

The question of the existence and location of a homolog in rats to the posterior association cortex in primates was discussed recently (Thomas \& Weir, 1975). It was suggested that one might argue that the rat had a homolog of primate association cortex and that Lashley's work (1941; see Figures 15 and 18) might be used as the guide to the locations of the parietal and temporal association areas in the rat. Thomas and Weir also presented evidence which confirmed Thomas' (1970) suggestion that Lashley's (1929) early data had provided questionable support for the equipotentiality of neocortical areas for maze III performance. Lesions in the parietal association area and medial frontal lesions resulted in significant performance decrements on maze III, whereas lesions of equivalent size in the frontopolar area did not.

The present work was intended to investigate further the possible functions of the proposed parietal and temporal association areas in the rat as well as to assess the generality of findings and conclusions concerning association vs. sensory cortex reached by Diamond and his associates using the tree shrew (Tupaia glis). To these ends, studies were done using (a) the Hebb-Williams mazes described by Rabinovitch and Rosvold, 1951, and (b) the "simple" and "complex" pattern discriminations and (c) the black-white discrimination reversal problems similar to those described and used by Diamond and his colleagues (Diamond \& Hall, 1969; Killacky, Snyder, \& Diamond, 1971).

Address requests for reprints to Mary G. Boyd, now at the Department of Psychology, College of Charleston, Charleston, South Carolina 29401.

\section{GENERAL METHODS}

\begin{abstract}
Subjects
Seventy-six male hooded rats (derived from the Simonsen Laboratories Long-Evans strain but bred here), ranging in age from 90 to 180 days at the beginning of the experiments, were used. The animals were housed individually and maintained on a light-dark cycle with light onset at 8:00 p.m. and offset at 8:00 a.m. local time. All testing was done during the dark phase. The rats were assigned randomly to the lesion and control groups and to the three experiments.
\end{abstract}

\section{Surgery and Histology}

Each experiment included groups with lesions in parietal and temporal cortex and a nonoperated control group. Additionally, each experiment included rats with either occipital or medial frontal cortical lesions. Intraperitoneal injections of Equithesin (.25 ml/100 g body weight) were given for surgical anesthesia. Open-drop ether was used as supplemental anesthesia when needed. Clean but nonsterile technique was used. No sign of infection was seen subsequent to the surgery.

When the animal reached a surgical level of anesthesia, a midline incision was made and the dorsal cranium was exposed. Lesions were produced by aspiration, and they were intended to approximate the magnitude of the trephine openings. Table 1 indicates the trephine sizes and lesion placements. Upon completion of the aspirations, the scalp was sutured, and the wound was dusted with tetracycline. The rat was then placed beneath a heat lamp to maintain normal body temperature until recovery from the anesthesia was complete.

At the end of the experiment, the animal was anesthesized with Equithesin and perfused through the dorsal aorta with $50 \mathrm{cc}$ of $0.9 \%$ saline followed by $50 \mathrm{cc}$ of $10 \%$ Formalin. After being placed in Formalin for a minimum of 3 days, the brains were photographed from the dorsal view with Polaroid continuous tone slide projection film, and the photographs were projected and adjusted to a 1:1 relationship with the dorsal view on a Lashley brain diagram. The lesion area was traced on the diagram and the percentage of cerebral surface occupied by the lesion was determined by the dot grid method (Thomas \& Peacock, 1965). Percentage assessments were not done for the medial frontal lesions as the medial surfaces of the rat brain apparently have never been included in the denominators used by those who have computed lesion percentages. Comparisons of lesion sizes 
Table 1

Diameters and Trephine Placements (in Millimeters) in Reference to the Coronal and Sagittal Sutures for the Five Cortical Lesions

\begin{tabular}{lccc}
\hline \multicolumn{1}{c}{ Lesion } & Diameter & Coronal* & Sagittal** \\
\hline Parietal & 3.5 & 1.0 & 1.0 \\
Temporal & 2.5 & 5.0 & 5.5 \\
Occipital & 2.5 & 7.5 & 1.0 \\
Striate & 3.5 & 7.5 & 2.0 \\
Medial Frontal & 5.5 & 2.8 & 0.0 \\
\hline
\end{tabular}

*All placements were caudal except the medial frontal, which was rostral to this suture.

**All placements were bilateral except the medial frontal, which was on the midline.

here with other reports in the literature were desired; therefore, the traditional means of computation was followed. Such a means of computation precludes a meaningful assessment of the medial frontal lesion percentages. Finally, the brains were embedded in celloidon and the areas including the lesions were sectioned at $40 \mu \mathrm{m}$, mounted on slides, and stained with thionin.

\section{EXPERIMENT 1}

Previous work has shown significant deficits on the Hebb-Williams closed-field test of rat intelligence (Rabinovitch \& Rosvold, 1951) by rats with lesions confined to the parietal area (Ain, Lubar, Moon, \& Kulig, 1969; Forgays, 1952; Thomas, 1966). However, Ain et al. did not report lesion sizes, and Forgays' and Thomas' studies were concerned with early $(2 \mathrm{~h})$ postoperative tests. Therefore, in addition to extending the investigation of performances on this task to a group with temporal lesions, it was useful to confirm earlier findings with parietal lesions. Following Forgays (1952) and Thomas (1966), a control group with occipital lesions was included.

\section{Method}

Subjects. Rats with parietal $(n=7)$, temporal $(n=7)$, occipital $(n=4)$, or no $(n=6)$ lesions were tested. Training began 14 days following surgery.

Apparatus and Procedure. The apparatus and procedures described by Rabinovitch and Rosvold (1951) were used with two exceptions: (a) Our animals were deprived of food $48 \mathrm{~h}$ prior to the first training session, as opposed to their recommended $10 \mathrm{~h}$, and (b) testing trials were limited to $2 \mathrm{~min}$. If an animal had not reached the goalbox in $2 \mathrm{~min}$, the errors accrued to that point were recorded and the next trial was begun. Briefly, the closed-field test of rat intelligence consists of mazelike problems. The 6 practice and 12 test problems are administered in succession one problem per day, 10 trials per problem.

\section{Results and Discussion}

Only the parietal group committed significantly more errors than the nonoperated group (see Table 2 ), as revealed by two-factor (Lesion/Nonlesion by Problems), with repeated measures on the problems factor analysis of variance, $F(3,20)=8.3, p<.01$, and the appropriate individual comparisons (Winer, 1962 , p. 378). None of the groups differed signifi- cantly on Problem 1 (possibly owing to its providing an unobstructed straight path from the startbox to the goalbox), but, as may be seen in Table 2 , the parietal group committed many more errors than any other group on Problems 2-12. The mean errors data for separate problems here compare favorably with those shown graphically by Ain et al. (1969). Estimating from their graph, their parietal group ranged from 30 errors on Problem 1 to more than 130 errors on Problem 12 and had mean errors scores greater than 70 on 8 of the 12 problems that they used.

Diagrams of the lesions may be seen in Figure 1. The reader should find good agreement between the loci of the parietal and temporal lesions in this experiment and the loci of these areas as mapped by Lashley (1941). For example, Lashley showed the parietal area of interest between anterior-posterior references lines 7 and 13. No parietal lesion here extended outside these lines. Similarly, Lashley showed the temporal area of interest between lines 11 and 15. Only one of our temporal lesions extended slightly beyond line 15 , and that only reached line 16 on one side. Some slight medial-to-lateral deviations from Lashley's maps occurred in both the parietal and temporal groups, perhaps owing to the irregular boundaries of these areas and our use of circular cranial openings. The differences in lesion sizes for the three groups were not significant (median test, $\left.\chi^{2}=2.42\right)$.

\section{EXPERIMENT 2}

Diamond and Hall (1969) and Killacky, Snyder, and Diamond (1971) compared the effects of striate and temporal lesions on "simple" and "complex" pattern discriminations. Since it appears to be accepted that striate lesions in rats preclude the ability to discriminate patterns (e.g., Lashley, 1931; Horel,

Table 2

Mean Errors on the 12 Problems of the Closed-Field Test of Rat Intelligence

\begin{tabular}{ccccc}
\hline & \multicolumn{4}{c}{ Groups } \\
\cline { 2 - 5 } Problems & Parietal & Temporal & Occipital & $\begin{array}{c}\text { Non- } \\
\text { operated }\end{array}$ \\
\hline 1 & 48 & 17 & 26 & 20 \\
2 & 103 & 7 & 16 & 21 \\
3 & 94 & 9 & 41 & 15 \\
4 & 130 & 8 & 28 & 11 \\
5 & 88 & 4 & 17 & 14 \\
6 & 127 & 7 & 21 & 6 \\
7 & 109 & 13 & 22 & 5 \\
8 & 130 & 8 & 14 & 4 \\
9 & 129 & 8 & 14 & 5 \\
10 & 105 & 8 & 11 & 3 \\
11 & 103 & 12 & 11 & 4 \\
12 & 124 & 8 & 5 & 3 \\
\hline
\end{tabular}



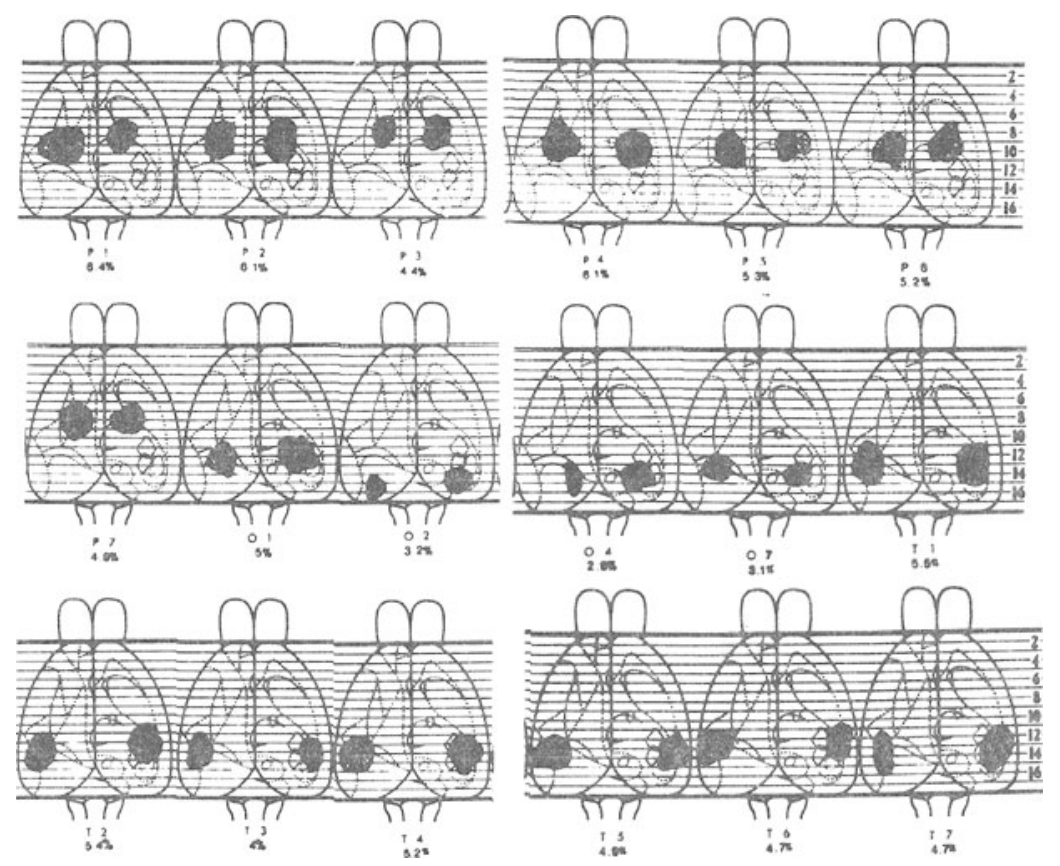

Figure 1. Lashley brain diagrams showing the extent of dorsal neocortical damage in the parietal (P), occipital (O), and temporal (T) lesion groups in Experiment 1.

Bettinger, Royce, \& Meyer, 1966; Thompson, 1969), it seemed meaningless to include a group with striate lesions here. In addition to the groups with parietal and temporal lesions, a group with medial frontal lesions was included. It was believed that such a group might provide a meaningful control as there is no reason to suspect that medial frontal lesions will disrupt pattern discrimination, and the medial frontal area in rats has been implicated as a possible homolog of primate frontal association cortex (Divac, 1971; Leonard, 1969).

\section{Method}

Subjects. Rats with parietal $(n=7)$, temporal $(n=7)$, or medial frontal $(n=7)$ lesions and nonoperated rats $(n=7)$ were trained on the simple and complex pattern discriminations beginning 14 days postoperatively.

Apparatus and Procedures. All animals were deprived of food for $48 \mathrm{~h}$ before being trained in a Yerkes-like discrimination apparatus similar to that described by Snyder and Diamond (1968). Beginning $25.5 \mathrm{~cm}$ from the startbox was a wall which reached the remaining $30 \mathrm{~cm}$ to the stimulus panels and divided the choice area into two compartments. When the rat left the startbox and entered the choice compartment, guillotine doors were closed to prevent retracing. Identical food cups were located in front of the stimulus panels, only one of which was baited (two 45-mg Noyes pellets) on a given trial. Behind the food cups were the stimulus panels. The stimuli were constructed from black and translucent white paper mounted on $10 \times 10 \mathrm{~cm}$ transparent Plexiglas panels. The panels were illuminated from behind by a $25-\mathrm{W}$ incandescent light bulb. The rats were adapted to the apparatus by being allowed to learn to eat the food pellets in the food cups in both goal compartments. Then they were allowed to explore the apparatus by being placed in the startbox with the door open. The doors to the goal compartments were also open, and both food cups were baited during the exploratory period. This adaptation procedure was repeated until it was judged that an animal had proceeded from the startbox to the goalboxes and had consumed the food pellets with minimal hesitation.

Stimuli for the simple discrimination were white equilateral triangles $(8 \mathrm{~cm}$ base) surrounded by a black field. The correct triangle was always upright (apex up) and the incorrect triangle was always inverted (apex down). The left-right positions of the correct stimulus were varied randomly. A correction procedure was used. If the incorrect compartment was entered, the rat was returned to the startbox and, with the positions of the stimuli unchanged, the trial was begun again. This procedure was repeated until the rat responded correctly; however, only the initial error was recorded. The rats were trained to a criterion of eight consecutive correct responses in a single session of 10 trials. Upon reaching criterion on the simple pattern discrimination, the animals were trained on the complex pattern discrimination. The stimuli were upright (positive) vs. inverted equilateral triangles $(8 \mathrm{~cm}$ base), each surrounded by an annulus (inside diameter, $8.8 \mathrm{~cm}$; outside diameter, $10.5 \mathrm{~cm}$ ). As before, the discriminanda were white against a black field. The same procedures and criterion were used.

\section{Results and Discussion}

Mean trials to criterion and mean errors for the four groups on the two pattern discrimination tasks may be seen in Table 3. On the simple pattern discrimination, the significant differences among the groups on trials to criterion, $\mathrm{F}(3,24)=5.94, \mathrm{p}<.01$, were shown by the Newman-Keuls procedure to be due to rats with temporal lesions taking more trials than the groups with parietal $(\mathrm{p}<.05)$ and medial frontal lesions $(p<.01)$ or the nonoperated group $(p<.01)$. The differences in mean errors, $F(3,24)$ $=3.93, \mathrm{p}<.05$, were due to deficits in the temporal group compared to the other groups (all $\mathrm{p}<.05$ ). 
Table 3

Mean Trials to Criterion and Mean Errors on the Simple and Complex Pattern Discrimination Tasks by the Parietal, Temporal, Medial Frontal, and Nonoperated Groups

\begin{tabular}{lcccc}
\hline & \multicolumn{4}{c}{ Groups } \\
\cline { 2 - 5 } & Parietal & Temporal & $\begin{array}{c}\text { Medial } \\
\text { Frontal }\end{array}$ & $\begin{array}{c}\text { Non- } \\
\text { operated }\end{array}$ \\
\hline & \multicolumn{4}{c}{ Simple Discrimination } \\
Trials & 80 & 99 & 73 & 76 \\
Errors & 38 & 50 & 36 & 36 \\
& \multicolumn{4}{c}{ Complex Discrimination } \\
Trials & 40 & 34 & 16 & 23 \\
Errors & 15 & 10 & 3 & 6 \\
\hline
\end{tabular}

None of the remaining groups differed on either measure.

On the complex pattern discrimination task, the parietal group required the most trials to criterion and committed the most errors. On trials to criterion, the mean differences among the groups, $F(3,24)$ $=6.55, \mathrm{p}<.01$, were revealed by the NewmanKeuls procedure to be due to differences between the parietal and nonoperated groups $(p<.05)$ and the parietal and medial frontal groups $(p<.01)$. The significant differences among the groups on errors on the complex discrimination, $F(3,24)=$ $7.27, \mathrm{p}<.01$, were shown by the Newman-Keuls procedure to be due to the parietal group's committing more errors than the nonoperated $(p<.05)$ and medial frontal $(p<.01)$ groups. The group with temporal lesions committed more errors than the group with medial frontal lesions $(p<.05)$.

The temporal lesions in this experiment (Figure 2) correspond closely to those in a report by Lashley and Frank (1932) which corrected an earlier conclusion (Lashley, 1931) that the area was essential to pattern vision. Lashley and Frank's rats were able to reach criterion on retention of an upright vs. inverted equilateral triangle discrimination problem. They suggested that the earlier report of failure was due to damage to the optic radiations. Both the temporal and the parietal lesions of this experiment corresponded well with the proposed association areas. The differences in lesion sizes for the parietal and temporal groups were not significant (MannWhitney $U=17)$. As noted earlier, it is not meaningful to compute the medial frontal lesion percentages. It may be noted that the medial frontal lesions were larger than the dorsal surface diagram might suggest, as the aspiration was intended to be deep along the medial walls.

Given the total failures in pattern discrimination, including the simple and complex patterns used here, following striate lesions in rats as established by Lashley (e.g., 1931) and given the deficits seen here following temporal lesions on the simple pattern discrimination, the present work together with Lashley's provides a partial extension of the generality of the findings reported by Diamond and Hall (1969) and Killacky et al., (1971) using tree shrews. Diamond and his colleagues reported deficits on the complex
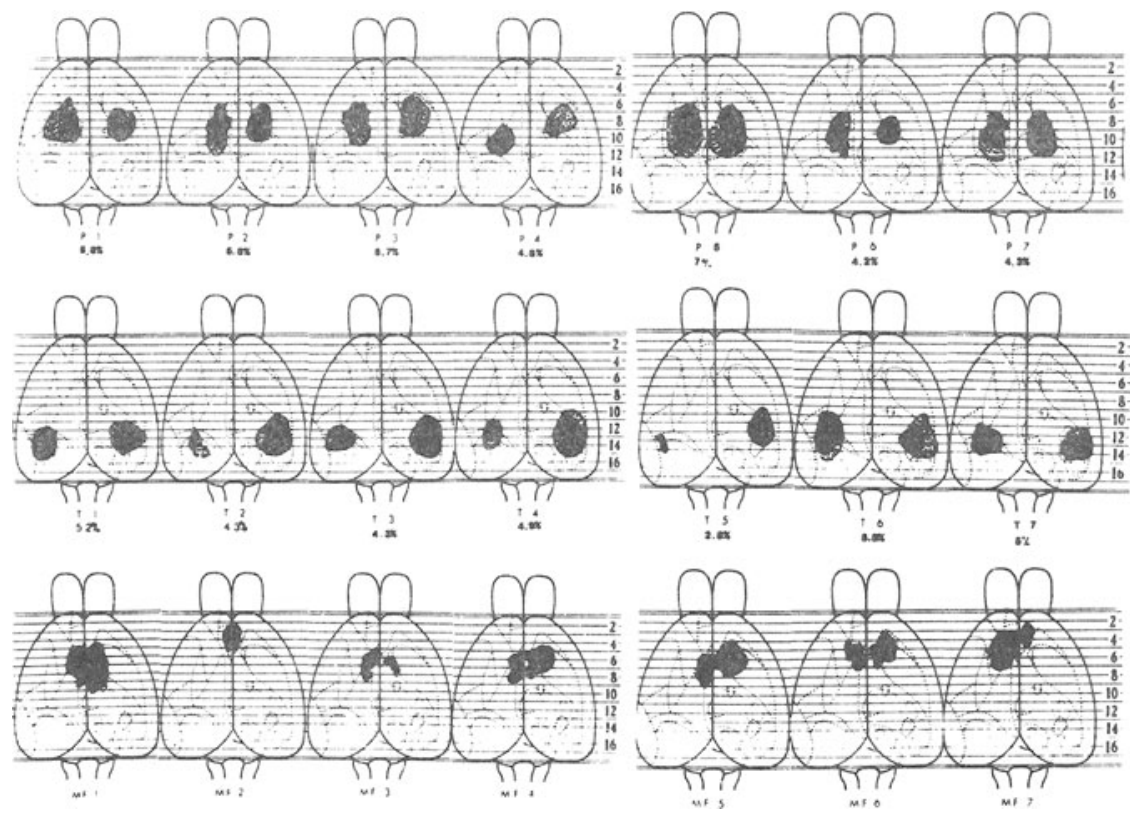

Figure 2. Lashley brain diagrams showing the extent of dorsal neocortical damage in the parietal (P), temporal (T), and medial frontal (MF) lesion groups of Experiment 2. 
but not on the simple pattern discriminations following striate lesions and relative but not total deficits on both discriminations following temporal lesions. Diamond and his colleagues did not examine the role of the parietal area in their studies, but the present work suggests that greater deficits may be seen following parietal than following temporal lesions.

\section{EXPERIMENT 3}

Contrary to the greater deficits seen following striate lesions than following temporal lesions on complex pattern discrimination, Killacky et al. (1971) reported greater deficits following temporal lesions than following striate lesions on black-white discrimination reversal learning by their tree shrews. The present experiment compared the effects of parietal, temporal, striate, and no lesions on blackwhite reversal learning.

\section{Method}

Subjects. Rats with parietal $(n=6)$, temporal $(n=6)$, striate $(n=6)$, or no $(n=6)$ lesions were trained, beginning 14 days postoperatively.

Apparatus and Procedures. The apparatus and the adaptation and correction procedures described in Experiment 2 were used. The discriminanda in the present work consisted of black or white paper mounted on $10 \times 10 \mathrm{~cm}$ Plexiglas panels. These were illuminated by a $25-\mathrm{W}$ bulb located to the rear and between the panels and by a $25-\mathrm{W}$ bulb mounted on the bottom-center of the table which held the apparatus. With positions of the stimulus panels being varied randomly, each animal was given 40 trials with both food cups baited to determine the animal's stimulus preference. Following these $\mathbf{4 0}$ trials, the nonpreferred stimulus was reinforced for that animal in the initial black-white discrimination (referred to in Results as a reversal owing to the reversal of the determined preference). Ten trials per session were administered, until the animal met a criterion of eight consecutive correct responses in a session. Following criterion and beginning the next day, the status of the reinforced and nonreinforced stimuli was reversed, and the animal was trained as before to the same criterion. A minimum of five reversals per animal was given. Unavoidable time constraints prevented running all groups through the same number of reversals. The decisions were made to terminate the striate group after five reversals, the parietal and nonoperated groups after seven reversals, and the temporal group after nine reversals.

\section{Results and Discussion}

As suggested in Figure 3, the nonoperated, parietal, and striate groups did not differ reliably through five reversals (Newman-Keuls ps were not significant), but the temporal group had more errors than any other group, $\mathrm{F}(3,20)=6.71, \mathrm{p}<.01$, and Newman-Keuls ps $<.01$. The significant difference across reversals, $\mathrm{F}(4,80)=55.8, \mathrm{p}<.01$, was due to the improvement shown by all groups, but Figure 3 suggests that the significant Groups by Reversals interaction, $F(12,80)=3.66, p<.01$, was due to the slower rate of improvement shown by the temporal group.

Diagrams of the lesions may be seen in Figure 4.

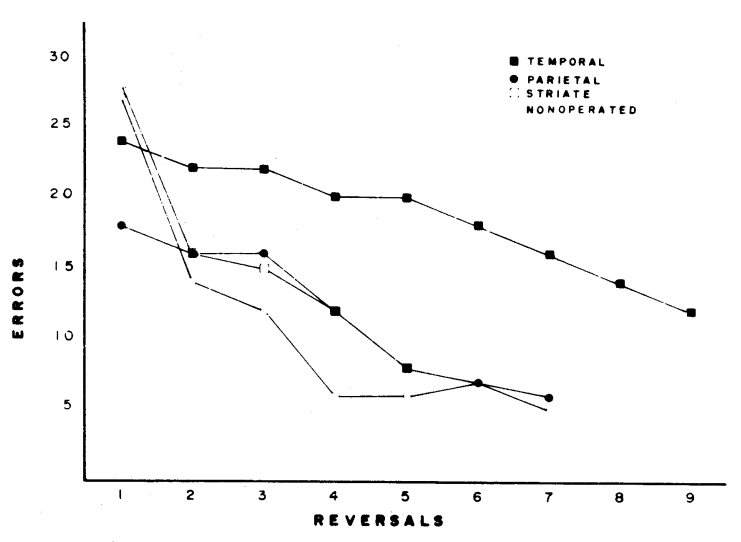

Figure 3. Mean errors as a function of successive black-white discrimination reversals.

There were significant differences among the groups in median lesion size (median test, $\chi^{2}=36$, $\mathrm{p}<.001)$. Specific comparisons using the MannWhitney statistic showed the temporal group to have lesions smaller than the parietal $(p=.032)$ or the striate groups $(p=.001)$. The parietal lesions were smaller than the striate lesions $(\mathrm{p}<.032)$. However, the difference between the parietal and striate lesions is of little concern, since those groups did not differ from nonoperated controls on the discrimination reversals. That the temporal group had the smallest lesions is also of minimal concern, since that group was the only one to show a deficit compared to the nonoperated controls.

The present study extends to the rat the generality of Killacky et al.'s (1971) finding, using tree shrews, that temporal lesions are more detrimental than striate lesions to improvements in performance on a series of black-white discrimination reversal problems. The deficits following temporal lesions seen here were not as profound as those reported by Killacky et al., but this difference may be attributed, in part, to their use of a more stringent criterion of performance and their more complete temporal lesions. Our median lesion (3.2\%) represents only $73 \%$ of the estimated size (we compute $4.4 \%$ ) of the temporal area of interest illustrated in Lashley's (1941) Figure 18. ${ }^{1}$

\section{GENERAL DISCUSSION}

The present work, together with a previous study from this laboratory (Thomas \& Weir, 1975), suggests a significant role for parietal cortex of the rat on performance using Lashley's maze III, the closed-field test of rat intelligence, and complex, but not simple, pattern discrimination. That the impaired performances seen on these tasks following parietal lesions is not due to some general traumatic effect is indicated by the normal performances seen on the 

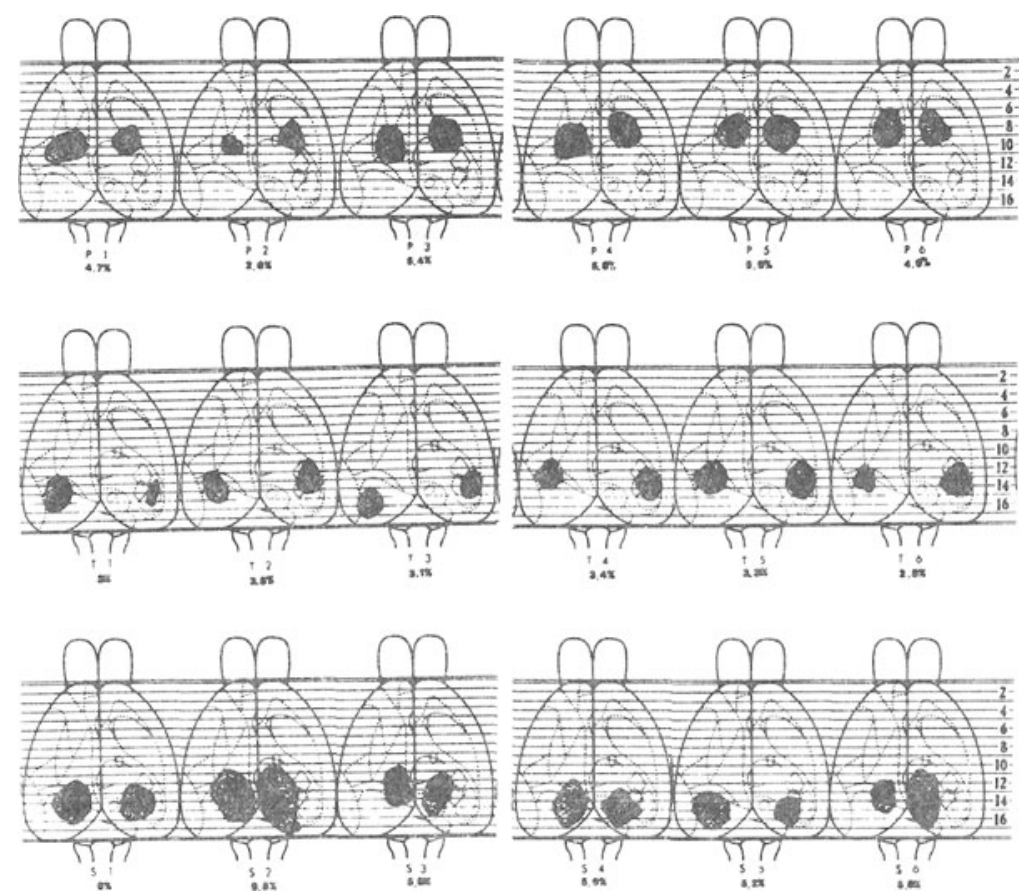

Figure 4. Lashley brain diagrams showing the extent of dorsal neocortical damage in the parietal (P), temporal (T), and striate (S) lesion groups of Experiment 3.

series of black-white discrimination reversals here and on a series of spatial reversals in an earlier study (Weir \& Thomas, 1975). A previous suggestion (Thomas \& Weir) that the parietal cortex in rats may be involved in spatial behavior obviously requires qualification, although it continues to merit consideration due to decrements in maze performances following parietal lesions and to the classic findings of Krechevsky (1935).

The impairment following parietal lesions on the "complex" but not the "simple" pattern discrimination compares to that reported by Diamond and Hall (1969) following lesions of the striate cortex in tree shrews. Apparently based on this finding, they concluded that "the geniculostriate system is not necessary for simple sensory tasks, but the striate cortex is required for a higher level of integration"' (p. 261). A similar conclusion might apply to the parietal cortex in rats, although a simpler explanation has not been eliminated. This simpler explanation assumes that the tree shrews with striate lesions or the rats with parietal lesions are impaired in their abilities to use brightness cues. However, this impairment is not deemed to be sufficient to prevent their learning the simple pattern discrimination on the basis of the brightness cues which result from looking at selected regions of the upright vs. inverted triangles (e.g., the upper or lower halves). The difficulty seen with the complex pattern discrimination is attributed to the reduction of brightness cues re- sulting from the addition of the annuli. Interestingly, Killacky et al. (1971) showed that tree shrews with striate lesions were able to learn the complex pattern discrimination following prolonged training with the discriminanda having only half-annuli. The prolonged training with the half-annuli might have enabled the shrews to learn to use the further reduced brightness cues which occur with complete annuli. Winans (1971) presented data to suggest the use of such brightness cues in a test comparable to the simple discrimination here by cats with striate lesions. In addition to measures such as those used by Winans (1971), assessments of the use of brightness cues in animals with parietal, temporal, or striate lesions might help to clarify the role of brightness as a cue for such animals in pattern discrimination problems.

The dissociation of effects following temporal lesions between deficits on the simple pattern discrimination and black-white reversals and normal performances on the complex pattern discrimination and on the maze of the closed-field test of intelligence argue against attributing the deficits to general traumatic effects. The diverse and dissociable deficits seen with rats having lesions in association cortex together with the comparability of our findings with those reported by Diamond and his colleagues using tree shrews suggest that meaningful questions about primate association cortex might be addressed in preliminary investigations using rats as models. To the 
extent that rats may serve as such models, significant advantages might be gained with respect to the conservation of primates. Additionally, the diverse and dissociable deficits seen here, considering also the relative smallness of the lesions, further question the acceptability of unqualified citations of the principles of mass function and equipotentiality in the context of Lashley's (1929) classic work. The majority of textbooks in physiological psychology published in this decade (see Thomas \& Weir, 1975, for earlier listing), including such recent ones as Brown (1976) and Leukel (1976), contain such unqualified citations.

\section{REFERENCE NOTE}

1. McDaniel, W. F., McDaniel, S., \& Thomas, R. K. Projections to the temporal cortex of the rat studied with two retrograde techniques. Paper presented at the meeting of the Southern Society for Philosophy and Psychology, Nashville, April 1977.

\section{REFERENCES}

Ain, B. R., Lubar, J. F., Moon, R. D., \& Kulug, B. M Effect of septal and neocortical damage on complex maze learning. Physiology and Behavior, 1969, 4, 235-238.

Brown, H. Brain and behavior. New York: Oxford University Press, 1976.

Diamond, I. T., \& Hall, W. C. Evolution of neocortex. Science, 1969, 164, 251-262.

Divac, I. Frontal lobe system and spatial reversal in the rat. Neuropsychologia, 1971, 9, 175-183.

Forgays, D. G. Reversible disturbances of function in rats following cortical insult. Journal of Comparative and Physiological Psychology, 1952, 45, 216-225.

Horel, J. A., Bettinger, L. A., Royce, C. J., \& Meyer, D. R. Role of neocortex in the learning and relearning of two visual habits by the rat. Journal of Comparative and Physiological Psychology, 1966, 61, 66-78.

Killacky, H., SNyder, M., \& Diamond, I. T. Function of striate and temporal cortex in the tree shrew. Journal of Comparative and Physiological Psychology Monograph, 1971, 74 (1, Pt. 2).

KRECHEVSKY, I. Brain mechanisms and "hypotheses." Journal of Comparative Psychology, 1935, 19, 425-468.

LASHLEY, K. S. Brain mechanisms and intelligence. Chicago: University of Chicago Press, 1929.

LASHLEY, K. S. The mechanism of vision IV. The cerebral areas necessary for pattern vision in the rat. Journal of Comparative Neurology, 1931, 53, 419-478.

LASHLEY, K. S. Thalamo-cortical connections of the rat's brain. Journal of Comparative Neurology, 1941, 75, 67-121.

LASHLEY; K. S., \& FranK, M. The mechanism of vision VI. The lateral portion of the area striata in the rat: A correction. Journal of Comparative Neurology, 1932, 55, 525-529.

LEONARD, C. M. The prefrontal cortex of the rat. I. Cortical projection of the mediodorsal nucleus. II. Efferent connections. Brain Research, 1969, 12, 321-343.

LEUKEL, F. Introduction to physiological psychology (3rd ed.). St. Louis: Mosby, 1976.

Rabinovitch, M. S., \& Rosvold, H. E. A closed-field intelligence test for rats. Canadian Journal of Psychology, 1951, 5, 122-128.

SNYDER, M., \& Diamond, I. T. The organization and function of the visual cortex in the tree shrew. Brain, Behavior and Evolution, 1968, 1, 244-288.

Tномаs, R. K. Immediate and subsequent effects of brain damage in rats. Journal of Comparative and Physiological Psychology, 1966, 62, 472-475.

Thomas, R. K. Mass function and equipotentiality: A reanalysis of Lashley's retention data. Psychological Reports, 1970, 27, 899-902.

Thomas, R. K., \& Peacock, L. J. A method for measuring brain lesions. Psychonomic Science, 1965, 3, 184.

Thomas, R. K., \& WeIR, V. K. The effects of lesions in the frontal or posterior association cortex of rats on maze III. Physiological Psychology, 1975, 3, 210-214.

Thompson, R. Localization of the "visual memory system" in the white rat. Journal of Comparative and Physiological Psychology Monograph, 1969, 69 (4, Pt. 2).

WEIR, V. K., \& Thomas, R. K. Failure to find spatial reversal deficits following medial frontal lesions. Bulletin of the Psychonomic Society, 1975, 5, 465-468.

Winans, S. S. Visual cues used by normal and visual-decorticate cats to discriminate figures of equal luminous flux. Journal of Comparative and Physiological Psychology, 1971, 74, 167-178.

WINER, B. J. Statistical principles in experimental design. New York: McGraw-Hill, 1962.

\section{NOTE}

1. Confirmation that the temporal cortical area shown in Lashley's Figure 18 (1941) is the recipient of projections from the lateral nucleus, pars posterior, was provided by McDaniel, McDaniel, and Thomas (Note 1), using Nissel and horseradish peroxidase methods. $\mathrm{McDaniel}$ et al. suggested that pars posterior may be the only site of thalamic projection to this temporal cortex and that the projections may only be ipsilateral.

(Received for publication April 22, 1977; revision accepted September 13, 1977.) 